\title{
Unification of the Quintuple and Septuple Product Identities
}

\author{
Wenchang Chu and Qinglun Yan* \\ Department of Applied Mathematics \\ Dalian University of Technology \\ Dalian 116024, P. R. China
}

Submitted: Jun 26, 2006; Accepted: Mar 20, 2007; Published: Mar 28, 2007

Mathematics Subject Classifications: 05A30, 14K25

\begin{abstract}
By combining the functional equation method with Jacobi's triple product identity, we establish a general equation with five free parameters on the modified Jacobi theta function, which can be considered as the common generalization of the quintuple, sextuple and septuple product identities. Several known theta function formulae and new identities are consequently proved.
\end{abstract}

\section{Introduction and Notation}

For two indeterminate $q$ and $x$ with $|q|<1$, the $q$-shifted factorial of infinite order reads as

$$
(x ; q)_{\infty}=\prod_{i=0}^{\infty}\left(1-x q^{i}\right)=(1-x)(1-q x)\left(1-q x^{2}\right) \cdots .
$$

Then we define the modified Jacobi theta function by

$$
\langle x ; q\rangle_{\infty}=(q ; q)_{\infty}(x ; q)_{\infty}(q / x ; q)_{\infty}
$$

\footnotetext{
${ }^{*}$ Email addresses: chu.wenchang@unile.it and yanqinglun@yahoo.com.cn
} 
Their product forms are abbreviated respectively as

$$
\begin{aligned}
(\alpha, \beta, \cdots, \gamma ; q)_{\infty} & =(\alpha ; q)_{\infty}(\beta ; q)_{\infty} \cdots(\gamma ; q)_{\infty} \\
\langle\alpha, \beta, \cdots, \gamma ; q\rangle_{\infty} & =\langle\alpha ; q\rangle_{\infty}\langle\beta ; q\rangle_{\infty} \cdots\langle\gamma ; q\rangle_{\infty}
\end{aligned}
$$

There are several important theta function identities in mathematical literature. Perhaps the simplest and the most significant one is Jacobi's triple product identity [23] (see [18, $\S 1.6]$ also):

$$
\langle x ; q\rangle_{\infty}=\sum_{n=-\infty}^{+\infty}(-1)^{n} q^{\left(\begin{array}{c}
n \\
2
\end{array}\right)} x^{n}
$$

For different proofs and applications, we refer the reader to the papers by Andrews [2], Chu [8], Ewell [10], Lewis [25], Mordell [27] and Wright [33]. The next one is the celebrated quintuple product identity attributed originally to Watson [32]. However, it can also be

found in Ramanujan's lost notebook, which has been cleared by Berndt [4, P 83]. Later in 1995, Ewell [11] found a sextuple product identity, which has both beautiful form and interesting applications to congruences of partition function (see Ewell $[12,13,14]$ ). Hirschhorn [21] found, first in 1983, the septuple product identity, which was subsequently rediscovered by Farkas and Kra [15] in 1999. More recently, Chapman [6], Foata-Han [16], Garvan [17] and Kongsirwong-Liu [24] provided different proofs. From algebraic point of view, some identities just mentioned can be recovered from the identities for affine root systems due to Macdonald [26] such as the quintuple product identity (from $B C_{1}$ ) and the septuple product identity (from $B C_{2}$ ), as observed in $[29, \S 6]$.

The purpose of the present paper is to unify all the identities just mentioned into a single formula. By means of the functional equation method and Jacobi's triple product identity, we shall show a general equation on the modified Jacobi theta function which covers several known and new theta function identities. The main theorem and its proof will be given in the next section. As applications, we shall systematically review the known identities such as the quintuple, sextuple and septuple products and establish several new theta function identities through Section 3 to 5 in the rest of the paper.

\section{Main Theorem and Proof}

By combining the functional equations with Jacobi's triple product identity, we prove the following fundamental result.

Theorem 1. Let $\alpha, \beta$ and $\gamma$ be three natural integers with $\operatorname{gcd}(\alpha, \gamma)=1$ and $\lambda=1+\alpha \beta^{2} \gamma$. 
For two indeterminate $x$ and $y$ with $x \neq 0$ and $y \neq 0$, there holds the algebraic identity:

$$
\begin{aligned}
\left\langle x ; q^{\alpha}\right\rangle_{\infty}\left\langle x^{\beta \gamma} y ; q^{\gamma}\right\rangle_{\infty}=\sum_{\ell=0}^{\alpha \beta^{2} \gamma}(-1)^{\ell} q^{\left(\begin{array}{l}
\ell \\
2
\end{array}\right) \alpha} x^{\ell} & \left\langle(-1)^{\alpha \beta} x^{\lambda} y^{\alpha \beta} q^{\left(\begin{array}{c}
\alpha \beta \\
2
\end{array}\right) \gamma+\ell \alpha} ; q^{\lambda \alpha}\right\rangle_{\infty} \\
& \times\left\langle(-1)^{\beta \gamma} y q^{\left(\begin{array}{c}
\beta \gamma+1 \\
2
\end{array}\right) \alpha-\ell \alpha \beta \gamma} ; q^{\lambda \gamma}\right\rangle_{\infty} .
\end{aligned}
$$

Proof. For the bivariate function $f(x, y)$ defined by the infinite product

$$
f(x, y)=\left\langle x ; q^{\alpha}\right\rangle_{\infty}\left\langle x^{\beta \gamma} y ; q^{\gamma}\right\rangle_{\infty}
$$

it is easy to see that $f(x, y)$ is analytic within $0<|x|<\infty$. Then we can expand it as a Laurent series in $x$ :

$$
f(x, y)=\sum_{k=-\infty}^{+\infty} \Omega_{k}(y) x^{k}
$$

From the definition of $f(x, y)$, it is trivial to check the functional equation

$$
f(x, y)=(-1)^{1+\alpha \beta} q^{\left(\begin{array}{c}
\alpha \beta \\
2
\end{array}\right) \gamma} x^{\lambda} y^{\alpha \beta} f\left(q^{\alpha} x, y\right),
$$

which corresponds to the recurrence relation

$$
\Omega_{k}(y)=(-1)^{1+\alpha \beta} y^{\alpha \beta} q^{(k-\lambda) \alpha+\left(\begin{array}{c}
\alpha \beta \\
2
\end{array}\right) \gamma} \Omega_{k-\lambda}(y) .
$$

Iterating this relation for $k$-times, we find that there exist $\lambda=1+\alpha \beta^{2} \gamma$ formal power series $\Omega_{\ell}(y)$ with $0 \leq \ell \leq \alpha \beta^{2} \gamma$ such that there hold

$$
\Omega_{k \lambda+\ell}(y)=(-1)^{k+k \alpha \beta} y^{k \alpha \beta} q^{\lambda\left(\begin{array}{c}
k \\
2
\end{array}\right) \alpha+k\left(\begin{array}{c}
\alpha \beta \\
2
\end{array}\right) \gamma+k \ell \alpha} \Omega_{\ell}(y) \quad \text { where } \quad k \in \mathbb{Z} .
$$

By invoking Jacobi's triple product identity, we can also expand $f(x, y)$ directly into the following double series:

$$
f(x, y)=\sum_{i=-\infty}^{+\infty} \sum_{j=-\infty}^{+\infty}(-1)^{i+j} q^{\left(\begin{array}{c}
i \\
2
\end{array}\right) \alpha+\left(\begin{array}{l}
j \\
2
\end{array}\right) \gamma} x^{i+j \beta \gamma} y^{j} .
$$

Equating the coefficients of $x^{\ell}$ in the two formal power series expansions of $f(x, y)$, we find that

$$
\begin{aligned}
\Omega_{\ell}(y) & =\sum_{j=-\infty}^{+\infty}(-1)^{j+\ell+j \beta \gamma} y^{j} q^{\left(\begin{array}{l}
\ell-j \beta \gamma \\
2
\end{array}\right) \alpha+\left(\begin{array}{l}
j \\
2
\end{array}\right) \gamma} \\
& =(-1)^{\ell} q^{\left(\begin{array}{l}
\ell \\
2
\end{array}\right) \alpha} \sum_{j=-\infty}^{+\infty}(-1)^{j+j \beta \gamma} y^{j} q^{\lambda\left(\begin{array}{l}
j \\
2
\end{array}\right) \gamma+j\left(\begin{array}{c}
\beta \gamma+1 \\
2
\end{array}\right) \alpha-j \ell \alpha \beta \gamma} .
\end{aligned}
$$


Therefore, $f(x, y)$ can be reformulated as

$$
\begin{aligned}
f(x, y) & =\sum_{\ell=0}^{\alpha \beta^{2} \gamma} \sum_{k=-\infty}^{+\infty} \Omega_{k \lambda+\ell}(y) x^{k \lambda+\ell}=\sum_{\ell=0}^{\alpha \beta^{2} \gamma}(-1)^{\ell} q^{\left(\begin{array}{l}
\ell \\
2
\end{array}\right) \alpha} x^{\ell} \\
& \times \sum_{k=-\infty}^{+\infty}(-1)^{k+k \alpha \beta} x^{k \lambda} y^{k \alpha \beta} q^{\lambda\left(\begin{array}{c}
k \\
2
\end{array}\right) \alpha+k\left(\begin{array}{c}
\alpha \beta \\
2
\end{array}\right) \gamma+k \ell \alpha} \\
& \times \sum_{j=-\infty}^{+\infty}(-1)^{j+j \beta \gamma} y^{j} q^{\lambda\left(\begin{array}{l}
j \\
2
\end{array}\right) \gamma+j\left(\begin{array}{c}
\beta \gamma+1 \\
2
\end{array}\right) \alpha-j \ell \alpha \beta \gamma} .
\end{aligned}
$$

Applying twice Jacobi's triple product identity to the double sum just displayed, we establish the equation stated in the theorem.

The simplest case $\alpha=\beta=\gamma=1$ of Theorem 1 reads as the following sextuple product identity, essentially discovered by Ewell $[11,12,14]$.

Corollary 2 (The sextuple product identity).

$$
\langle x, x y ; q\rangle_{\infty}=\left\langle-q y,-x^{2} y ; q^{2}\right\rangle_{\infty}-x\left\langle-y,-q x^{2} y ; q^{2}\right\rangle_{\infty} .
$$

In fact, making the parameter replacements $q \rightarrow q^{2}, x \rightarrow-q x y$ and $y \rightarrow 1 / y^{2}$ and then applying Jacobi's triple product identity, we see that the last equation is equivalent to the sextuple product identity due to Ewell [12, Eq 2.2]:

$$
\begin{aligned}
\left\langle-q x y,-q x / y ; q^{2}\right\rangle_{\infty} & =\sum_{i=-\infty}^{+\infty} q^{2 i^{2}} x^{2 i} \sum_{j=-\infty}^{+\infty} q^{2 j^{2}} y^{2 j} \\
& +q \sum_{i=-\infty}^{+\infty} q^{2 i(i+1)} x^{2 i+1} \sum_{j=-\infty}^{+\infty} q^{2 j(j+1)} y^{2 j+1}
\end{aligned}
$$

If we let $q \rightarrow q^{2}, x \rightarrow-q$ and $y \rightarrow 1$ in Corollary 2 , then we can get immediately another identity due to Ewell [13, Thm 2.1]:

$$
\prod_{n=1}^{\infty}\left(1-q^{4 n-2}\right)^{2}\left(1+q^{2 n-1}\right)^{4}=\prod_{n=1}^{\infty}\left(1+q^{4 n-2}\right)^{4}+4 q \prod_{n=1}^{\infty}\left(1+q^{4 n}\right)^{4} .
$$

For more applications of the sextuple product identity, the interested reader may consult Ewell [11, 12, 13, 14]. 


\section{The Quintuple Product Identities}

This section reviews the application of Theorem 1 to quintuple product identities. We first state the case $\beta=1$ of Theorem 1 as follows.

Proposition 3. Let $\alpha$ and $\gamma$ be two natural integers with $\operatorname{gcd}(\alpha, \gamma)=1$. For two indeterminate $x$ and $y$ with $x \neq 0$ and $y \neq 0$, there holds the algebraic identity:

$$
\begin{aligned}
\left\langle x ; q^{\alpha}\right\rangle_{\infty}\left\langle x^{\gamma} y ; q^{\gamma}\right\rangle_{\infty}=\sum_{\ell=0}^{\alpha \gamma}(-1)^{\ell} q^{\left(\begin{array}{l}
\ell \\
2
\end{array}\right) \alpha} & x^{\ell} \\
& \left\langle(-1)^{\alpha} x^{1+\alpha \gamma} y^{\alpha} q^{\left(\begin{array}{c}
\alpha \\
2
\end{array}\right) \gamma+\ell \alpha} ; q^{\alpha(1+\alpha \gamma)}\right\rangle_{\infty} \\
& \times\left\langle(-1)^{\gamma} y q^{\left(\begin{array}{c}
\gamma+1 \\
2
\end{array}\right) \alpha-\ell \alpha \gamma} ; q^{\gamma(1+\alpha \gamma)}\right\rangle_{\infty} .
\end{aligned}
$$

When $\alpha=2$ and $\gamma=1$, we find from Proposition 3 the following generalized quintuple product identity.

Corollary 4.

$$
\begin{aligned}
\langle x ; q\rangle_{\infty}\left\langle x y ; q^{2}\right\rangle_{\infty} & =\left\langle-q y ; q^{3}\right\rangle_{\infty} \sum_{i=-\infty}^{+\infty}(-1)^{i} q^{3 i^{2}-2 i} x^{3 i} y^{i} \\
& -\left\langle-q^{3} y ; q^{3}\right\rangle_{\infty} \sum_{i=-\infty}^{+\infty}(-1)^{i} q^{3 i^{2}} x^{3 i+1} y^{i+1} \\
& +\left\langle-q^{2} y ; q^{3}\right\rangle_{\infty} \sum_{i=-\infty}^{+\infty}(-1)^{i} q^{3 i^{2}+2 i} x^{3 i+2} y^{i+1}
\end{aligned}
$$

Instead, when $\alpha=1$ and $\gamma=2$, we recover from Proposition 3 another generalized quintuple product identity.

\section{Corollary 5 (Hirschhorn [22, Eq 2]).}

$$
\begin{aligned}
\langle x ; q\rangle_{\infty}\left\langle x^{2} y ; q^{2}\right\rangle_{\infty} & =\left\langle q^{3} y ; q^{6}\right\rangle_{\infty} \sum_{i=-\infty}^{+\infty} q^{3\left(\begin{array}{c}
i \\
2
\end{array}\right)} x^{3 i} y^{i} \\
& -\left\langle q y ; q^{6}\right\rangle_{\infty} \sum_{i=-\infty}^{+\infty} q^{3\left(\begin{array}{c}
i \\
2
\end{array}\right)+i} x^{3 i+1} y^{i} \\
& -\left\langle q^{5} y ; q^{6}\right\rangle_{\infty} \sum_{i=-\infty}^{+\infty} q^{3\left(\begin{array}{c}
i \\
2
\end{array}\right)+2 i} x^{3 i+2} y^{i+1}
\end{aligned}
$$

We remark that under the parameter replacement $y \rightarrow x y$, the left member displayed in Corollary 4 becomes that in Corollary 5 . However the corresponding right members are not euqivalent. 
For $x \rightarrow-q x$ and $y \rightarrow-q^{-1}$, Corollary 5 reduces to the following identity due to Stanton $[30,1986]$, who derived it through sign variations of the Macdonald identities.

Example 6 (Stanton [30]).

$$
\begin{aligned}
\langle-q x ; q\rangle_{\infty}\left\langle-q x^{2} ; q^{2}\right\rangle_{\infty} & =\left\langle-1 ; q^{6}\right\rangle_{\infty} \sum_{i=-\infty}^{+\infty} q^{3\left(\begin{array}{c}
i \\
2
\end{array}\right)+3 i+1} x^{3 i+1} \\
& +\left\langle-q^{2} ; q^{6}\right\rangle_{\infty} \sum_{i=-\infty}^{+\infty} q^{3\left(\begin{array}{c}
i \\
2
\end{array}\right)+2 i}\left\{1+q^{2 i+1} x^{2}\right\} x^{3 i}
\end{aligned}
$$

Letting $y=1$ and $y=q$ in Corollary 5, we further get respectively the following two quintuple product identities.

\section{Example 7.}

$$
\begin{aligned}
\langle x ; q\rangle_{\infty}\left\langle x^{2} ; q^{2}\right\rangle_{\infty} & =\left\langle q^{3} ; q^{6}\right\rangle_{\infty} \sum_{i=-\infty}^{+\infty} q^{3\left(\begin{array}{c}
i \\
2
\end{array}\right)} x^{3 i} \\
& -\left\langle q ; q^{6}\right\rangle_{\infty} \sum_{i=-\infty}^{+\infty} q^{3\left(\begin{array}{c}
i \\
2
\end{array}\right)+i}\left(1+q^{i} x\right) x^{3 i+1}
\end{aligned}
$$

Example 8 (The quintuple product identity).

$$
\langle x ; q\rangle_{\infty}\left\langle q x^{2} ; q^{2}\right\rangle_{\infty}=\left(q^{2} ; q^{2}\right)_{\infty} \sum_{i=-\infty}^{+\infty} q^{3\left(\begin{array}{c}
i \\
2
\end{array}\right)+i}\left(1-x q^{i}\right) x^{3 i}
$$

The last one is the so-called quintuple product identity. For the historical note, different proofs and extensions about this celebrated identity, the reader can refer to $[1,3,5,19$, $22,31]$. A comprehensive bibliography for the proofs of this identity is given in Cooper's survey paper [9]. In addition, Paule [28] found a finite form of the quintuple product identity. Two further finite forms have been given in the recent papers [7] and [20].

\section{The Septuple Product Identities}

In order to derive the generalized septuple product identities, we state first the case $\beta=2$ of Theorem 1 as follows. 
Proposition 9. Let $\alpha$ and $\gamma$ be two natural integers with $\operatorname{gcd}(\alpha, \gamma)=1$. For two indeterminate $x$ and $y$ with $x \neq 0$ and $y \neq 0$, there holds the algebraic identity:

$$
\begin{aligned}
\left\langle x ; q^{\alpha}\right\rangle_{\infty}\left\langle x^{2 \gamma} y ; q^{\gamma}\right\rangle_{\infty}=\sum_{\ell=0}^{4 \alpha \gamma}(-1)^{\ell} q^{\left(\begin{array}{l}
\ell \\
2
\end{array}\right) \alpha} & x^{\ell} \\
& \left\langle x^{1+4 \alpha \gamma} y^{2 \alpha} q^{\left(\begin{array}{c}
2 \alpha \\
2
\end{array}\right) \gamma+\ell \alpha} ; q^{\alpha(1+4 \alpha \gamma)}\right\rangle_{\infty} \\
& \left.\times y q^{\left({ }^{2 \gamma+1} 2\right) \alpha-2 \ell \alpha \gamma} ; q^{\gamma(1+4 \alpha \gamma)}\right\rangle_{\infty} .
\end{aligned}
$$

When $\alpha=\gamma=1$, replacing $q$ by $q^{2}$ in Proposition 9 leads to the following generalized septuple product identity.

\section{Corollary 10.}

$$
\begin{aligned}
\left\langle x, x^{2} y ; q^{2}\right\rangle_{\infty} & =\left\langle q^{6} y ; q^{10}\right\rangle_{\infty} \sum_{i=-\infty}^{+\infty}(-1)^{i} q^{5 i^{2}-3 i} x^{5 i} y^{2 i} \\
& -\left\langle q^{2} y ; q^{10}\right\rangle_{\infty} \sum_{i=-\infty}^{+\infty}(-1)^{i} q^{5 i^{2}-i} x^{5 i+1} y^{2 i} \\
& -\left\langle q^{8} y ; q^{10}\right\rangle_{\infty} \sum_{i=-\infty}^{+\infty}(-1)^{i} q^{5 i^{2}+i} x^{5 i+2} y^{2 i+1} \\
& +\left\langle q^{4} y ; q^{10}\right\rangle_{\infty} \sum_{i=-\infty}^{+\infty}(-1)^{i} q^{5 i^{2}+3 i} x^{5 i+3} y^{2 i+1} \\
& -\left\langle y ; q^{10}\right\rangle_{\infty} \sum_{i=-\infty}^{+\infty}(-1)^{i} q^{5 i^{2}+5 i+2} x^{5 i+4} y^{2 i+1}
\end{aligned}
$$

The two particular cases of interest may be displayed as follows.

Example 11 (The septuple product identity: $x \rightarrow x$ and $y \rightarrow 1$ in Corollary 10).

$$
\begin{aligned}
\left\langle x, x^{2} ; q^{2}\right\rangle_{\infty} & =\left\langle q^{4} ; q^{10}\right\rangle_{\infty} \sum_{i=-\infty}^{+\infty}(-1)^{i} q^{5 i^{2}-3 i}\left\{1+q^{6 i} x^{3}\right\} x^{5 i} \\
& -\left\langle q^{2} ; q^{10}\right\rangle_{\infty} \sum_{i=-\infty}^{+\infty}(-1)^{i} q^{5 i^{2}-i}\left\{1+q^{2 i} x\right\} x^{5 i+1}
\end{aligned}
$$

We point out that this septuple product identity first appeared in Hirschhorn [21] explicitly, even though it has been attributed erroneously to Farkas-Kra [15] by Foata-Han [16, Eq 1.6] and Chapman [6, Thm 1]. 
Example 12 (Kongsiriwong-Liu [24, Eq 7.36]: $x \rightarrow q x$ and $y \rightarrow q^{-1}$ in Corollary 10).

$$
\begin{aligned}
\left\langle q x, q x^{2} ; q^{2}\right\rangle_{\infty} & =\left\langle q^{5} ; q^{10}\right\rangle_{\infty} \sum_{i=-\infty}^{+\infty}(-1)^{i} q^{5 i^{2}} x^{5 i} \\
& -\left\langle q ; q^{10}\right\rangle_{\infty} \sum_{i=-\infty}^{+\infty}(-1)^{i} q^{5 i^{2}+2 i+1}\left\{1-q^{6 i+3} x^{3}\right\} x^{5 i+1} \\
& -\left\langle q^{3} ; q^{10}\right\rangle_{\infty} \sum_{i=-\infty}^{+\infty}(-1)^{i} q^{5 i^{2}+4 i+1}\left\{1-q^{2 i+1} x\right\} x^{5 i+2}
\end{aligned}
$$

\section{$5 \quad$ Further Theta Function Identities}

For the presence of five free parameters, we may further specialize Theorem 1 to numerous theta function identities. Leaving the parameter $\gamma$ invariant, this section collects four theta function identities, just for examples, which have appeared previously in Kongsiriwong-Liu [24]. Here all the parameter settings refer to Theorem 1 under the same base substitution $q \rightarrow q^{2}$.

Example $13\left(\left[24\right.\right.$, Thm 6]: $\alpha=1$ and $\beta=1: x \rightarrow q x$ and $\left.y \rightarrow q^{-\gamma}\right)$.

$$
\begin{aligned}
\left\langle q x ; q^{2}\right\rangle_{\infty}\left\langle x^{\gamma} ; q^{2 \gamma}\right\rangle_{\infty}=\sum_{\ell=0}^{\gamma}(-1)^{\ell} q^{\ell^{2}} & \left\{\sum_{i=-\infty}^{+\infty}(-1)^{(\gamma+1) i} q^{\left(\gamma^{2}+\gamma\right) i^{2}-(2 \ell+1) \gamma i}\right\} \\
& \times\left\{\sum_{j=-\infty}^{+\infty} q^{(\gamma+1) j^{2}+(2 \ell-\gamma) j} x^{(\gamma+1) j+\ell}\right\} .
\end{aligned}
$$

Example $14([24$, Thm 7]: $\alpha=1$ and $\beta=1: x \rightarrow q x$ and $y \rightarrow 1)$.

$$
\begin{aligned}
\left\langle q x ; q^{2}\right\rangle_{\infty}\left\langle q^{\gamma} x^{\gamma} ; q^{2 \gamma}\right\rangle_{\infty}=\sum_{\ell=0}^{\gamma}(-1)^{\ell} q^{\ell^{2}} & \left\{\sum_{i=-\infty}^{+\infty}(-1)^{(\gamma+1) i} q^{\left(\gamma^{2}+\gamma\right) i^{2}-2 \ell \gamma i}\right\} \\
\times & \left\{\sum_{j=-\infty}^{+\infty} q^{(\gamma+1) j^{2}+2 \ell j} x^{(\gamma+1) j+\ell}\right\} .
\end{aligned}
$$

Example 15 ([24, Thm 8]: $\alpha=1$ and $\beta=2: x \rightarrow x$ and $y \rightarrow 1)$.

$$
\begin{aligned}
\left\langle x ; q^{2}\right\rangle_{\infty}\left\langle x^{2 \gamma} ; q^{2 \gamma}\right\rangle_{\infty}=\sum_{\ell=0}^{4 \gamma}(-1)^{\ell} q^{\ell^{2}-\ell} & \left\{\sum_{i=-\infty}^{+\infty}(-1)^{i} q^{\left(4 \gamma^{2}+\gamma\right) i^{2}+(1-4 \ell) \gamma i}\right\} \\
\times & \left.\times \sum_{j=-\infty}^{+\infty}(-1)^{j} q^{(4 \gamma+1) j^{2}+(2 \ell-2 \gamma-1) j} x^{(4 \gamma+1) j+\ell}\right\} .
\end{aligned}
$$


Example 16 ([24, Thm 9]: $\alpha=1$ and $\beta=2: x \rightarrow q x$ and $\left.y \rightarrow q^{-\gamma}\right)$.

$$
\begin{aligned}
\left\langle q x ; q^{2}\right\rangle_{\infty}\left\langle q^{\gamma} x^{2 \gamma} ; q^{2 \gamma}\right\rangle_{\infty}=\sum_{\ell=0}^{4 \gamma}(-1)^{\ell} q^{\ell^{2}} & \left\{\sum_{i=-\infty}^{+\infty}(-1)^{i} q^{\left(4 \gamma^{2}+\gamma\right) i^{2}-4 \ell \gamma i}\right\} \\
\times & \left\{\sum_{j=-\infty}^{+\infty}(-1)^{j} q^{(4 \gamma+1) j^{2}+2 \ell j} x^{(4 \gamma+1) j+\ell}\right\} .
\end{aligned}
$$

\section{References}

[1] K. Alladi, The quintuple product identity and shifted partition functions, J. Comput. Appl. Math. 68 (1996), 3-13.

[2] G. E. Andrews, A simple proof of Jacobi's triple product identity, Proc. Amer. Math. Soc. 16 (1965), 333-334.

[3] A. O. L. Atkin and P. Swinnerton-Dyer, Some properties of partitions, Proc. London Math. Soc. 4 (1954), 84-106.

[4] B. C. Berndt, Ramanujan's Notebooks, Part III, Springer-Verlag, New York, 1991.

[5] L. Carlitz and M. V. Subbarao, A simple proof of the quintuple product identity, Proc. Amer. Math. Soc. 32:1 (1972), 42-44.

[6] R. Chapman, On a septuple product identity, The Andrews Festschrift (Maratea, 1998); Actes $42^{e}$ Séminaire Lotharingien de Combinatoire 43 (1999), 1-4.

[7] W. Y. C. Chen, W. Chu and N. S. S. Gu, Finite form of the quintuple product identity, J. Combin. Theory (Ser. A) 113:1 (2006), 185-187.

[8] W. Chu, Durfee rectangles and the Jacobi triple product identity, Acta Math. Sinica (New Series) 9:1 (1993), 24-26.

[9] S. Cooper, The quintuple product identity, International Journal of Number Theory 2:1 (2006), 115-161.

[10] J. A. Ewell, An easy proof of the triple-product identity, Amer. Math. Monthly 88 (1981), 270-272.

[11] J. A. Ewell, Arithmetical consequences of a sextuple product identity, Rocky Mountain J. Math. 25 (1995), 1287-1293.

[12] J. A. Ewell, On an identity of a Ramanujan, Proc. Amer. Math. Soc. 125 (1997), 3769-3771.

[13] J. A. Ewell, A note on a Jacobian identity, Proc. Amer. Math. Soc. 126 (1998), 421423.

[14] J. A. Ewell, Further consequences of a sextuple product identity, Rocky Mountain J. Math. 32 (2002), 123-128.

[15] H. M. Farkas and I. Kra, On the quintuple product identity, Proc. Amer. Math. Soc. 127:3 (1999), 771-778. 
[16] D. Foata and G. N. Han, The triple, quintuple and septuple product identities revisited, The Andrews Festschrift (Maratea, 1998); Actes $42^{e}$ Séminaire Lotharingien de Combinatoire 42 (1999), 1-12.

[17] F. G. Garvan, A generalization of the Hirschhorn-Farkas-Kra septagonal numbers identity, Discrete math. 232 (2001), 113-118 .

[18] G. Gasper and M. Rahman, Basic Hypergeometric Series (2nd edition), Cambridge University Press, 2004.

[19] B. Gordon, Some identities in combinatorial analysis, Quart. J. Math. (Oxford) 12 (1961), 285-290.

[20] V. J. W. Guo and J. Zeng, Short proofs of summation and transformation formulas for basic hypergeometric series, J. Math. Anal. Appl. 327:1 (2007), 310-325.

[21] M. D. Hirschhorn, A simiple proof of an identity of Ramanujan, J. Austral. Math. Soc. (Ser. A) 34 (1983), 31-35.

[22] M. D. Hirschhorn, A generalisation of the quintuple product identity, J. Austral. Math. Soc. (Ser. A) 44 (1988), 42-45.

[23] C. G. J. Jacobi, Fundamenta Nova Theoriae Functionum Ellipticarum, Fratrum Bornträger Regiomonti, 1829; Gesammelte werke, Erster Band, G. Reimer, Berlin, 1881.

[24] S. Kongsiriwong and Z. G. Liu, Uniform proofs of q-series-product identities, Results in Math. 44 (2003), 312-339. .

[25] R. P. Lewis, A combinatorial proof of the triple product identity, Amer. Math. Monthly 91 (1984), 420-423.

[26] I. G. Macdonald, Affine root systems and Dedekinds $\eta$-function, Invent. Math. 15 (1972), 91-143.

[27] L. J. Mordell, An identity in combinatorial analysis, Proc. Glasgow Math. Ass. 5 (1961), 197-200.

[28] P. Paule, Short and easy computer proofs of the Rogers-Ramanujan identities and of identities of similar type, Electron. J. Combin. 1 (1994), R\#10.

[29] H. Rosengren and M. Schlosser, Elliptic determinant evaluations and the Macdonald identities for affine root systems, Compositio Math. 142 (2006), 937-961.

[30] D. Stanton, Sign variations of the Macdonald identities, SIAM. J. Math. Annal. 17(1986), 1454-1460.

[31] M. V. Subbarao and M. Vidyasagar, On Watson's quintuple product identity, Proc. Amer. Math. Soc. 26:1 (1970), 23-27.

[32] G. N. Watson, Theorems stated by Ramanujan VII: Theorems on continued fractions, J. London Math. Soc. 4 (1929), 39-48.

[33] E. M. Wright, An enumerative proof of an identity of Jacobi, J. London Math. Soc. 40 (1965), 55-57. 